\title{
THE NATURE OF THE RED-SHIFTS IN QUASI-STELLAR OBJECTS
}

\author{
L. WOLTJER \\ (Dept. of Astronomy, Columbia University, New York, N.Y., U.S.A.)
}

Interpretations of the red-shifts are reviewed.

(1) The gravitational interpretation of red-shifts encounters three difficulties:

(a) If a mass $M$ is surrounded by emitting gas, the volume appears to be too small to explain the forbidden-line emission.

(b) Narrow lines imply a stable, thin, spherical sheet of gas in a strong gravitational field.

(c) The magnitude of the red-shifts is too large.

Fowler and Hoyle proposed a very dense galactic nucleus with $10^{13}$ neutron stars in a region of 1 parsec diameter. This may get around (b) and (c), but (a) is still difficult.

(2) Kinematical red-shifts can be associated with either large velocities of objects ejected from many galaxies scattered through the universe, or from our galaxy alone. The first possibility leads to a preponderance of blue-shifts, which are not observed; the second, to a total energy far larger than that involved in most strong radio galaxies. Our galaxy is only a quite weak radio source.

(3) The cosmological interpretation of the red-shift meets with the following objections:

(a) There is no clear magnitude-red-shift relation, but large-amplitude variability of individual objects leads us to expect only a weak relation.

(b) Time variations are incompatible with synchrotron radiation, but this is not the case if the relativistic electrons move outward more or less radially. Also, the synchrotron nature of the variable component is not completely certain.

(c) Large energies are involved, but the situation is no worse than that for radio galaxies.

Support for the cosmological interpretation comes from:

(d) NGC 1275, which contains an unresolved radio component with a variable behavior very similar to that of quasi-stellar objects.

(e) The angular separations of the double radio sources associated with some quasistellars, which indicate linear separations comparable with those in radio galaxies. 